\title{
AN APPLICATION OF TOPOLOGICAL DEGREE TO THE PERIODIC COMPETING SPECIES PROBLEM
}

\author{
CARLOS ALVAREZ ${ }^{1}$ AND ALAN C. LAZER ${ }^{2}$
}

(Received 11 November 1985)

\begin{abstract}
We consider the Volterra-Lotka equations for two competing species in which the right-hand sides are periodic in time. Using topological degree, we show that conditions recently given by $\mathrm{K}$. Gopalsamy, which imply the existence of a periodic solution with positıve components, also imply the uniqueness and asymptotic stability of the solution. We also give optimal upper and lower bounds for the components of the solution.
\end{abstract}

\section{Introduction}

The topological degree of a mapping has long been known to be a useful tool for establishing the existence of fixed points of nonlinear mappings. The main purpose of this paper is to show how the most basic properties of degree can be used to establish the uniqueness, in a certain region, of the fixed point of a mapping connected with a problem from mathematical biology.

Our main motivation comes from two recent papers of Gopalsamy [8], [9].

We consider the system of differential equations

$$
\begin{aligned}
& u^{\prime}(t)=u(t)[a(t)-b(t) u-c(t) v] \\
& v^{\prime}(t)=v(t)[d(t)-e(t) u-f(t) v]
\end{aligned}
$$

where the functions $a(t), \ldots, f(t)$ are continuous, positive functions of the time $t \in(-\infty, \infty)$ and are assumed to be periodic with common period $T>0$. This system models the competition between two species in a $T$-periodic environment ([6], [8], [12]). We are interested in the existence of $T$-periodic solutions $\operatorname{col}(u(t), v(t))$ with both components positive. (Here $\operatorname{col}(\xi, \eta)$ denotes the column

\footnotetext{
${ }^{1}$ Departamento de Matematicas, Facultad de Ciencias, Universidad de Los Andes, Merida, Venezuela

${ }^{2}$ Department of Mathematics and Computer Science, University of Miami, Coral Gables, Fla., 33124, U.S.A.

(C) Copyright Australian Mathematical Society 1986, Serial-fee code 0334-2700/86
} 
vector with components $\xi$ and $\eta$ ). In case $a(t), \ldots, f(t)$ are positive constants $a, \ldots, f$, it has long been known that a necessary and sufficient condition that there exist an equilibrium point $\operatorname{col}\left(u_{0}, v_{0}\right)$, with $u_{0}>0$ and $v_{0}>0$, which attracts all solutions with initial values in the open first quadrant of the $u$, $v$-plane is that

$$
a>c d / f, \quad d>e a / b .
$$

(see, for example [13]).

In [8], K. Gopalsamy gave a partial extension of this result to the nonautonomous periodic case. To state Gopalsamy's result, we introduce the following notations: If $g$ is a continuous $T$-periodic function defined on $(-\infty, \infty)$, we set

$$
g_{M}=\max _{t} g(t), \quad g_{L}=\min _{t} g(t) .
$$

In [8], it was assumed that the growth rates $a(t)$ and $d(t)$ were continuous, positive and $T$-periodic and that $b(t), c(t), e(t)$, and $f(t)$ were positive constants $b, c, e$, and $f$ respectively. Using results due to Krasnoselskii concerning monotone operators which are strictly positive and concave with respect to cones, it was shown that the conditions

$$
a_{L}>c d_{M} / f, \quad d_{L}>e a_{M} / b
$$

imply the existence of an open rectangle $\Omega$ in the first quadrant of the $u, v$-plane and a $T$-periodic solution $\operatorname{col}\left(u_{0}(t), v_{0}(t)\right)$ of $(1.1)$ such that $\left(u_{0}(t), v_{0}(t)\right) \in \Omega$ for all $t$, and all solutions of (1.1) with initial values in $\Omega$ tend to $\operatorname{col}\left(u_{0}(t), v_{0}(t)\right)$ as $t \rightarrow \infty$.

Recently in [9], Gopalsamy considered the system

$$
u_{i}^{\prime}(t)=u_{t}(t)\left[a_{i}(t)-\sum_{j=1}^{n} c_{t j}(t) u_{j}(t)\right]
$$

for $i=1, \ldots, n$ where the functions $a_{i}, c_{i j}(i, j=1, \ldots, n)$ are continuous, positive, and $T$-periodic. In [9] it was shown that the conditions

$$
a_{i L}>\sum_{\substack{j=1 \\ j \neq i}}^{n} c_{\imath, M} a_{j M} / c_{j j L}
$$

for $i=1, \ldots, n$ imply the existence of a $T$-periodic solution $\operatorname{col}\left(u_{10}(t), \ldots, u_{n 0}(t)\right)$ of (1.2) all of whose components are positive. In [9] it was also shown that if the inequalities (1.3) hold and if, in addition,

$$
a_{J j L}>\sum_{\substack{i=1 \\ i \neq j}}^{n} a_{i j M}, \quad 1 \leqslant j \leqslant n,
$$

then (1.2) has a unique $T$-periodic solution with all components positive, and this solution is asymptotically stable and attracts all solutions whose components have positive initial values. 
For the two-dimensional system (1.1), the conditions (1.3) and (1.4) become

$$
a_{L}>c_{M} d_{M} / f_{L}, \quad d_{L}>e_{M} a_{M} / b_{L}
$$

and

$$
b_{L}>e_{M}, \quad f_{L}>c_{M} .
$$

Clearly the conditions (1.5) and (1.6) are independent, even in the constant coefficient case. For example, if $a=3, b=c=2, d=f=1$ and $e=1 / 3$, then the inequalities (1.5) hold, but the second inequality in (1.6) does not hold.

The major part of this paper is to show how the topological degree can be used to prove that the conditions (1.5) alone imply the existence of a unique T-periodic solution of (1.1) whose components are positive, which is asymptotically stable and which attracts all solutions with components which are initially positive. This is done in three steps: First, it is shown that if $F: \mathbf{R}^{2} \rightarrow \mathbf{R}^{2}$ is the time $T$-map or Poincaré map associated with (1.1) and $I$ is the identity map, then there are numbers $k_{1}>0$ and $k_{2}>0$ such that for all sufficiently small $\delta>0$, the degree of the mapping $I-F$ with respect to the open rectangle $\left\{(\xi, \eta) \mid \delta<\xi<k_{1}, \delta<\eta<\right.$ $\left.k_{2}\right\}$ and the point $(0,0)$ is equal to 1 . Next, it is shown that if $\operatorname{col}\left(u_{0}(t), v_{0}(t)\right)$ is any $T$-periodic solution of (1.1) with both components positive, then the characteristic multipliers $\alpha_{1}$ and $\alpha_{2}$ associated with this periodic solution satisfy $0<\alpha_{1}<1$ and $0<\alpha_{2}<1$. This implies that such a solution is locally asymptotically exponentially stable, and that the Jacobian of $I-F$ at the fixed point of $F$ corresponding to this solution is $\left(1-\alpha_{1}\right)\left(1-\alpha_{2}\right)$. Since this implies that the local index of $I-F$ at a fixed point of $F$ in the rectangle described above is equal to 1 , by a basic result of degree theory, there is a unique fixed pont of $F$ in the rectangle. Finally, the uniqueness of the fixed point in the rectangle is shown to imply that the corresponding $T$-periodic solution attracts all solutions having both components positive at the same time.

In [12] de Mottoni and Schiaffino considered the system (1.1) where the functions $a(t), \ldots, f(t)$ were assumed to be continuous and $T$-periodic but only $b(t), c(t), e(t)$ and $f(t)$ were assumed to be positive for all $t$. It was assumed that the averages of $a(t)$ and $d(t)$ were positive. It was shown by de Mottoni and Schiaffino that there exist unique positive $T$-periodic functions $p(t)$ and $q(t)$ such that

$$
p^{\prime}(t)+a(t) p(t)=b(t), \quad q^{\prime}(t)+d(t) q(t)=f(t) .
$$

If one sets $x(t)=p(t) u(t) y(t)=q(t) v(t)$ where $\operatorname{col}(u(t), v(t))$ is a solution of (1.1) then

$$
\begin{aligned}
& x^{\prime}(t)=x(t) G(t)[1-x(t)-g(t) y(t)] \\
& y^{\prime}(t)=y(t) H(t)[1-y(t)-h(t) x(t)]
\end{aligned}
$$


where

$$
\begin{aligned}
G(t) & =b(t) / p(t), & H(t) & =f(t) / q(t), \\
g(t) & =\frac{p(t) c(t)}{b(t) q(t)}, & h(t) & =\frac{q(t) e(t)}{f(t) p(t)} .
\end{aligned}
$$

Studying the system (1.8), de Mottoni and Schiaffino used a theorem proved in [12] to show that if $g(t)<1$ and $h(t)<1$, then there is a unique $T$-periodic stable solution of (1.8), with both components positive, which attracts all solutions which start in the open first quadrant.

To show that the theorem of de Mottoni and Schiaffino does not include the one stated above, we consider the following example of (1.1):

$$
\begin{aligned}
& u^{\prime}=u[1-(2+\cos t) u-0.9 v] \\
& v^{\prime}=v[1-0.9 u-v]
\end{aligned}
$$

Here $a(t)=d(t)=f(t) \equiv 1, c(t)=e(t) \equiv 0.9, b(t)=2+\cos t$. It follows that

$$
\begin{aligned}
& a_{L}-c_{M} d_{M} / f_{L}=1-0.9>0, \\
& d_{L}-e_{M} a_{M} / b_{L}=1-0.9>0,
\end{aligned}
$$

so conditions (1.5) are satisfied. The unique, positive, $T$-periodic functions $p(t)$ and $q(t)$ such that

$$
\begin{aligned}
& 0=p^{\prime}(t)+a(t) p(t)-b(t) \\
& 0=q^{\prime}(t)+d(t) q(t)-f(t)
\end{aligned}
$$

are

$$
p(t)=2+(1 / 2)(\cos t+\sin t), \quad q(t) \equiv 1 .
$$

Therefore, if $g(t)=p(t) c(t) / q(t) b(t)$, then $g(\pi / 2)=9 / 8>1$ so the conditions of de Mottoni and Schiaffino fail.

Through elementary means, we show that if conditions (1.5) hold, then the unique $T$-periodic $\operatorname{col}\left(u_{0}(t), v_{0}(t)\right)$ of $(1.1)$ with $u_{0}(t)>0$ and $v_{0}(t)>0$ for all $t$ satisfies

$$
\begin{aligned}
& \frac{a_{L} f_{L}-c_{M} d_{M}}{b_{M} f_{L}-e_{L} c_{M}} \leqslant u_{0}(t) \leqslant \frac{a_{M} f_{M}-c_{L} d_{L}}{b_{L} f_{M}-e_{M} c_{L}}, \\
& \frac{b_{L} d_{L}-e_{M} a_{M}}{b_{L} f_{M}-e_{M} c_{L}} \leqslant v_{0}(t) \leqslant \frac{b_{M} d_{M}-e_{L} a_{L}}{b_{M} f_{L}-e_{L} c_{M}},
\end{aligned}
$$

for all $t$. Obviously, these bounds are optimal, as can be seen by considering the autonomous case. Recently our colleague, Shair Ahmad has considered the system (1.1) where the functions $a(t), \ldots, f(t)$ are merely assumed to be continuous and bounded above and below by positive constants on an interval of the form $t_{0} \leqslant t<\infty$. 
Using different methods, he has shown that if conditions (1.5) hold, where $g_{L}$ and $g_{M}$ denote inf $g(t)$ and $\sup g(t)$ respectively for $t \geqslant t_{0}$, then (1.5) has a solution $\operatorname{col}\left(u_{0}(t), v_{0}(t)\right)$ whose components have the same upper and lower bounds given above for $t_{0} \leqslant t<\infty$.

In the final section we show our main result concerning the system (1.1) can be used to improve a result due to $C$. Cosner and the second author in [4], which deals with a model which allows spatial dependence and diffusion.

\section{Comparison theorems}

Let us assume that the functions $a(t), \ldots, f(t)$ appearing in (1.1) are continuous, positive, and $T$-periodic. If $u(t)$ is a nonnegative solution $u^{\prime}(t)=u(t)[a(t)$ $-b(t) u(t)]$ and $v(t)$ is a nonnegative solution of $v^{\prime}(t)=v(t)[d(t)-f(t) v(t)]$, then both $\operatorname{col}(u(t), 0)$ and $\operatorname{col}(0, v(t))$ are solutions of the system (1.1). From the uniqueness theorem, it follows that the closed first quadrant in the $(u, v)$ plane is invariant with respect to the system (1.1), in the sense that if $\operatorname{col}(u(t), v(t))$ is a solution with $u_{0}(t) \geqslant 0$ for some $t_{0}$, then $u(t) \geqslant 0$ and $v(t) \geqslant 0$ on the domain of $\operatorname{col}(u(t), v(t))$. The same argument shows that the open first quadrant is also invariant.

LEMMA 2.1. If $\operatorname{col}\left(u_{k}(t), v_{k}(t)\right)$ is a solution of (1.1) for $k=1,2$, with $u_{1}(0) \geqslant$ $u_{2}(0) \geqslant 0$ and $v_{2}(0) \geqslant v_{1}(0) \geqslant 0$, then $u_{1}(t) \geqslant u_{2}(t), v_{2}(t) \geqslant v_{1}(t)$ for all $t \in$ $[0, \infty)$ as long as both solutions $\operatorname{col}\left(u_{1}(t), v_{2}(t)\right), \operatorname{col}\left(u_{2}(t), v_{2}(t)\right)$ are defined. The above inequalities are strict if $u_{1}(0)>u_{2}(0)$ and $v_{2}(0)>v_{1}(0)$.

Proof. Since solutions of (1.1) are continuous with respect to initial conditions, it suffices to prove the result under the stronger assumptions

$$
u_{1}(0)>u_{2}(0)>0, \quad v_{2}(0)>v_{1}(0)>0 .
$$

By continuity, the inequalities

$$
u_{1}(t)>u_{2}(t), \quad v_{2}(t)>v_{1}(t)
$$

will hold for $t$ sufficiently small and positive. If (2.1) does not hold for all $t \geqslant 0$ in the domain of $\operatorname{col}\left(u_{k}(t), v_{k}(t)\right) k=1,2$, there exists $\bar{t}>0$ such that (2.1) holds for $0 \leqslant t<\bar{t}$ and either: (a) $u_{1}(\bar{t})=u_{2}(\bar{t})$ or (b) $v_{1}(\bar{l})=v_{2}(\bar{i})$. Suppose (a) holds. By continuity we must have $v_{1}(\bar{t}) \leqslant v_{2}(\bar{l})$, and so, by the uniqueness theorem for differential equations, $v_{1}(\bar{t})<v_{2}(\bar{t})$. By invariance of the open first quadrant, $u_{1}(\bar{t})=u_{2}(\bar{t})>0$. Since $u_{1}(t)-u_{2}(t)>0$ on $[0, \bar{t})$ we must have $u_{1}^{\prime}(\bar{i})-u_{2}^{\prime}(\bar{i}) \leqslant 0$. On the other hand, from (1.1) we have that

$$
u_{1}^{\prime}(\bar{i})-u_{2}^{\prime}(\bar{t})=u_{1}(\bar{t}) c(\bar{t})\left[v_{2}(\bar{t})-v_{1}(\bar{t})\right]>0 \text {. }
$$


This contradiction shows that case (a) is impossible and a similar argument proves the same for case (b). By previous remarks, the lemma is proved.

Given a continuous $T$-periodic function $g(t)$ defined on $(-\infty, \infty)$ we let $g_{M}$ denote the maximum value of $g(t)$ and $g_{L}$ denote the minimum value of $g(t)$. In addition to assuming that the functions $a(t), \ldots, f(t)$ are continuous, positive, and $T$-periodic we shall assume in the following that

$$
\begin{aligned}
& a_{L}>c_{M} d_{M} / f_{L}, \\
& d_{L}>e_{M} a_{M} / b_{L} .
\end{aligned}
$$

In the following $k_{1}$ and $k_{2}$ will denote numbers such that $k_{1}>a_{M} / b_{L}$, $k_{2}>d_{M} / f_{L}$ and $d_{L}>e_{M} k_{1}, a_{L}>c_{M} k_{2}$.

Let $\delta>0$ be chosen so $\delta<k_{1}, \delta<k_{2}$, and

$$
\begin{aligned}
& a_{L}-c_{M} k_{2}-b_{M} \delta>0, \\
& d_{L}-e_{M} k_{1}-f_{M} \delta>0 .
\end{aligned}
$$

LEMma 2.2. If $\operatorname{col}\left(u^{*}(t), v_{*}(t)\right)$ and $\operatorname{col}\left(u_{*}(t), v^{*}(t)\right)$ denote the solutions of (1.1) which satisfy the initial conditions $u^{*}(0)=k_{1}, v_{*}(0)=\delta$

$$
u_{*}(0)=\delta, v^{*}(0)=k_{2},
$$

then

$$
\begin{aligned}
& u_{*}(t)<u^{*}(t), \quad v_{*}(t)<v^{*}(t), \\
& u^{*}(0)>u^{*}(t)>u^{*}(t+T), \\
& v_{*}(0)<v_{*}(t)<v_{*}(t+T), \\
& u_{*}(0)<u_{*}(t)<u_{*}(t+T), \\
& v_{*}(0)>v_{*}(t)>v_{*}(t+T),
\end{aligned}
$$

for all $t>0$.

Proof. Since $u^{*}(0)>u_{*}(0)$ and $v^{*}(0)>v_{*}(0),(2.6)$ is a consequence of Lemma 2.1. We have

$$
\begin{aligned}
d u^{*}(0) / d t & =u^{*}(0)\left[a(0)-b(0) u^{*}(0)-c(0) v_{*}(0)\right] \\
& <u^{*}(0)\left[a(0)-b(0) a_{M} / b_{L}\right] \leqslant 0
\end{aligned}
$$

and from (2.5)

$$
\begin{aligned}
d v_{*}(0) / d t & =v_{*}(0)\left[d(0)-e(0) u^{*}(0)-f(0) v_{*}(0)\right] \\
& \geqslant v_{*}(0)\left[d_{L}-e_{M} k_{1}-f_{M} \delta\right]>0 .
\end{aligned}
$$

Similarly, $d v^{*}(0) / d t<0$, and from (2.7) we infer that $d u_{*}(0) / d t>0$. From what has been shown, it follows that for $t$ sufficiently small and positive

$$
u^{*}(0)>u^{*}(t), \quad v_{*}(0)<v_{*}(t) .
$$


If, contrary to the claim, these inequalities did not hold for all $t>0$, there would exist $\bar{i}>0$ such that $(2.11)$ holds on $[0, \bar{t})$ and either: (a) $u^{*}(\bar{t})=u^{*}(0)$ or (b) $v_{*}(\bar{i})=v_{*}(0)$. If (b) held, then $u^{*}(\bar{t}) \leqslant u^{*}(0)=k_{1}$ and $v_{*}^{\prime}(\bar{t}) \leqslant 0$. But

$$
\begin{aligned}
v_{*}^{\prime}(\bar{t}) & =v_{*}(\bar{t})\left[d(\bar{t})-e(\bar{t}) u^{*}(\bar{t})-f(\bar{t}) v_{*}(\bar{t})\right] \\
& \geqslant v_{*}(0)\left[d_{L}-e_{M} k_{1}-f_{M} \delta\right]>0
\end{aligned}
$$

so we have a contradiction. Similarly, if (a) held, then $v_{*}(\bar{t}) \geqslant v_{*}(0)>0$ and we have the contradictory inequalities

$$
\begin{aligned}
d u^{*}(\bar{t}) / d t & \geqslant 0 \text { and } \\
d u^{*}(\bar{t}) / d t & =u^{*}(\bar{t})\left[a(\bar{t})-b(\bar{t}) u^{*}(\bar{t})-c(\bar{t}) v_{*}(\bar{t})\right] \\
& <u^{*}(\bar{t})\left[a(\bar{t})-b(\bar{t}) a_{M} / b_{L}\right] \leqslant 0
\end{aligned}
$$

so (a) is impossible. Therefore, both inequalities in (2.11) hold for all $t>0$.

By $T$-periodicity of $a(t), \ldots, f(t)$ we see that

$$
\operatorname{col}\left(u_{1}^{*}(t), v_{1 *}(t)\right) \equiv \operatorname{col}\left(u^{*}(t+T), v_{*}(t+T)\right)
$$

is a solution of (1.1). By (2.11), $u^{*}(0)>u^{*}(T)=u_{1}^{*}(0)$ and $v_{*}(0)<v_{*}(T)=$ $v_{1 *}(0)$. Therefore, by Lemma 2.1, we see that $u^{*}(t)>u_{1}^{*}(t)=u^{*}(t+T)$,

$$
v_{*}(t)<v_{1 *}(t)=v_{*}(t+T),
$$

for all $t>0$. Together with (2.11), this proves (2.7) and (2.8).

The proofs of (2.9) and (2.10) which make use of (2.4) are entirely similar and therefore omitted.

Given $(r, s) \in \mathbf{R}^{2}$ we let $\operatorname{col}(u(t, r, s), v(t, r, s))$ denote the solution of (1.1) such that $u(0, r, s)=r, v(0, r, s)=s$.

By the uniqueness theorem of differential equations and the $T$-periodicity of the system (1.1) in $t, u(t+T, r, s) \equiv u(t, r, s), v(t+T, r, s) \equiv v(t, r, s)$ if and only $u(T, r, s)=r, v(T, r, s)=s$. For this reason we study the mapping $F$ : $\mathbf{R}^{2} \rightarrow \mathbf{R}^{2}$ defined by

$$
F(r, s)=(r-u(T, r, s), s-v(T, r, s)) .
$$

LEMMA 2.3. If $D$ denotes the open rectangle in the $r$, s-plane consisting of points $(r, s)$ such that $\delta<r<k_{1}, \delta<s<k_{2}$, then $\operatorname{deg}(F, D, \overline{0})=1$ where $\operatorname{deg}(F, D, \overline{0})$ denotes the Brouwer degree of $F$ with respect to $D$ and $\overline{0}=(0,0)$.

Proof. Let $\operatorname{col}\left(u^{*}(t), v_{*}(t)\right)$ and $\operatorname{col}\left(u_{*}(t), v^{*}(t)\right)$ be the solutions of (1.1) defined in Lemma 2.2. If

$$
\begin{aligned}
& u_{*}(0)=\delta \leqslant r \leqslant k_{1}=u^{*}(0), \\
& v_{*}(0)=\delta \leqslant s \leqslant k_{2}=v^{*}(0),
\end{aligned}
$$


then, according to Lemma 2.1 and Lemma 2.2, we have

$$
\delta=u_{*}(0)<u_{*}(T) \leqslant u(T, r, s) \leqslant u^{*}(T)<u^{*}(0)=k_{1}
$$

and $\delta=v_{*}(0)<v_{*}(T) \leqslant v(T, r, s) \leqslant v^{*}(T)<v^{*}(0)=k_{2}$. This shows that the mapping $(r, s) \rightarrow(u(T, r, s), v(T, r, s))$ maps the closure of $D$ into $D$. Let $\left(r_{1}, s_{1}\right)$ be a definite point in $D$. Since $D$ is convex, it follows that if $(r, s)$ belongs to the boundary of $D$, then for all $\lambda \in[0,1]$

$$
\left(r_{1}+\lambda\left(u(T, r, s)-r_{1}\right), s_{1}+\lambda\left(v(T, r, s)-s_{1}\right)\right) \equiv N(r, s, \lambda) \in D .
$$

Consequently, $(r, s)-N(r, s, \lambda) \neq \overline{0}$ for $(r, s) \in \partial D$ and $\lambda \in[0,1]$. By homotopy invariance of degree (see, for example [11] or [14]), it follows that the degrees of the two mappings $(r, s) \rightarrow(r, s)-N(r, s, 1)=F(r, s)$ and $(r, s) \rightarrow(r, s)-$ $N(r, s, 0)=\left(r-r_{1}, s-s_{1}\right)$ with respect to $D$ and $\overline{0}$ are the same. Since $\left(r-r_{1}, s\right.$ $\left.-s_{1}\right)=(0,0)$ has the unique solution $(r, s)=\left(r_{1}, s_{1}\right)$ in $D$ and the Jacobian of the mapping $(r, s) \rightarrow\left(r-r_{1}, s-s_{1}\right)$ is identically equal to 1 , it follows that its degree with respect to $D$ and $\overline{0}$ is 1 . This proves the lemma.

\section{The local index}

The purpose of this section is to prove

Lemma 3.1. Let $F$ and $D$ be as in Lemma 2.3. If $\left(r_{0}, s_{0}\right) \in D$ and $F\left(r_{0}, s_{0}\right)=$ $(0,0)$ then the Jacobian of $F$ at $(0,0)$ is positive.

Proof. The Jacobian of $F$ at $\left(r_{0}, s_{0}\right)$ is equal to the determinant

$$
\left|\begin{array}{cc}
1-\frac{\partial u}{\partial r}\left(T, r_{0}, s_{0}\right) & -\frac{\partial u}{\partial s}\left(T, r_{0}, s_{0}\right) \\
-\frac{\partial v}{\partial r}\left(T, r_{0}, s_{0}\right) & 1-\frac{\partial v}{\partial s}\left(T, r_{0}, s_{0}\right)
\end{array}\right| .
$$

Therefore, if $\alpha_{1}$ and $\alpha_{2}$ are the eigenvalues of the matrix

$$
\left[\begin{array}{ll}
\frac{\partial u}{\partial r}\left(T, r_{0}, s_{0}\right) & \frac{\partial u}{\partial s}\left(T, r_{0}, s_{0}\right) \\
\frac{\partial v}{\partial r}\left(T, r_{0}, s_{0}\right) & \frac{\partial v}{\partial s}\left(T, r_{0}, s_{0}\right)
\end{array}\right],
$$

then the Jacobian of $F$ at $\left(r_{0}, s_{0}\right)$ is equal to $\left(1-\alpha_{1}\right)\left(1-\alpha_{2}\right)$. We shall prove that $0<\alpha_{1}<1$ and $0<\alpha_{2}<1$ and from this the lemma will follow.

If

$$
\begin{aligned}
& g_{1}(u, v, t)=u[a(t)-b(t) u-c(t) v] \\
& g_{2}(u, v, t)=v[d(t)-e(t) u-f(t) v]
\end{aligned}
$$


then $\operatorname{col}(u(t, r, s), v(t, r, s))$ is the solution of the system

$$
\begin{aligned}
& u^{\prime}(t)=g_{1}(t, u(t), v(t)), \\
& v^{\prime}(t)=g_{2}(t, u(t), v(t)),
\end{aligned}
$$

which satisfies the initial condition $u(0)=r, v(0)=s$. It follows from the basic theorem concerning differentiability of solutions with respect to initial conditions (see, for example, $[10$, p. $21-23]$ ) that if

$$
X(t)=\left[\begin{array}{ll}
\frac{\partial u}{\partial r}\left(t, r_{0}, s_{0}\right) & \frac{\partial u}{\partial s}\left(t, r_{0}, s_{0}\right) \\
\frac{\partial v}{\partial r}\left(t, r_{0}, s_{0}\right) & \frac{\partial v}{\partial s}\left(t, r_{0}, s_{0}\right)
\end{array}\right]
$$

then

$$
X^{\prime}(t)=A(t) X(t), \quad X(0)=I
$$

where

$$
A(t)=\left[\begin{array}{ll}
\frac{\partial g_{1}}{\partial u}\left(t, u\left(t, r_{0}, s_{0}\right), v\left(t, r_{0}, s_{0}\right)\right) & \frac{\partial g_{1}}{\partial v}\left(t, u\left(t, r_{0}, s_{0}\right), v\left(t, r_{0}, s_{0}\right)\right) \\
\frac{\partial g_{2}}{\partial u}\left(t, u\left(t, r_{0}, s_{0}\right), v\left(t, r_{0}, s_{0}\right)\right) & \frac{\partial g_{2}}{\partial v}\left(t, u\left(t, r_{0}, s_{0}\right), v\left(t, r_{0}, s_{0}\right)\right)
\end{array}\right]
$$

and $I$ is the identity matrix.

If for brevity, we set $u\left(t, r_{0}, s_{0}\right)=u_{0}(t), v\left(t, r_{0}, s_{0}\right)=v_{0}(t)$, then

$$
A(t)=\left[\begin{array}{lr}
a(t)-2 b(t) u_{0}(t)-c(t) v_{0}(t) & -c(t) u_{0}(t) \\
-e(t) v_{0}(t) & d(t)-e(t) u_{0}(t)-2 f(t) v_{0}(t)
\end{array}\right]
$$

Since $u_{0}(0)=r_{0}>0, v_{0}(0)=s_{0}>0$ and the open first quadrant in the $u, v$-plane is invariant with respect to (1.1), we have $u_{0}(t)>0, v_{0}(t)>0$ for all $t$. Moreover, since $F\left(r_{0}, s_{0}\right)=(0,0), u_{0}(t+T) \equiv u_{0}(t)$ and $v_{0}(t+T) \equiv v_{0}(t)$.

Let $x(t)=\operatorname{col}\left(x_{1}(t), x_{2}(t)\right)$ be a solution of the vector system $x^{\prime}(t)=A(t) x(t)$. Since

$$
\begin{aligned}
u_{0}^{\prime}(t) / u_{0}(t) & =a(t)-b(t) u_{0}(t)-c(t) v_{0}(t) \\
v_{0}^{\prime}(t) / v_{0}(t) & =d(t)-e(t) u_{0}(t)-f(t) v_{0}(t)
\end{aligned}
$$

we see that

$$
\begin{aligned}
& x_{1}^{\prime}(t)=\frac{u_{0}^{\prime}(t)}{u_{0}(t)} x_{1}(t)-b(t) u_{0}(t) x_{1}(t)-c(t) u_{0}(t) x_{2}(t) \\
& x_{2}^{\prime}(t)=\frac{v_{0}^{\prime}(t)}{v_{0}(t)} x_{2}(t)-e(t) v_{0}(t) x_{1}(t)-f(t) v_{0}(t) x_{2}(t)
\end{aligned}
$$


Rewriting these equations in the form

$$
\begin{aligned}
{\left[x_{1}^{\prime}(t) u_{0}(t)\right.} & \left.-u_{0}^{\prime}(t) x_{1}(t)\right] / u_{0}(t)^{2} \\
& =-b(t) u_{0}(t) x_{1}(t) / u_{0}(t)-c(t) v_{0}(t) x_{2}(t) / v_{0}(t), \\
{\left[x_{2}^{\prime}(t) v_{0}(t)\right.} & \left.-v_{0}^{\prime}(t) x_{2}(t)\right] / v_{0}(t)^{2} \\
& =-e(t) u_{0}(t) x_{1}(t) / u_{0}(t)-f(t) v_{0}(t) x_{2}(t) / v_{0}(t),
\end{aligned}
$$

it follows that if $y(t)=\operatorname{col}\left(x_{1}(t) / u_{0}(t), x_{2}(t) / v_{0}(t)\right)$, then $y^{\prime}(t)=B(t) y(t)$, where

$$
B(t)=\left[\begin{array}{ll}
-b(t) u_{0}(t) & -c(t) v_{0}(t) \\
-e(t) u_{0}(t) & -f(t) v_{0}(t)
\end{array}\right] .
$$

Since each column of the matrix $X(t)$ is a solution of the vector differential equation $x^{\prime}(t)=A(t) x(t)$, we see that if

$$
P(t)=\left[\begin{array}{ll}
1 / u_{0}(t) & 0 \\
0 & 1 / v_{0}(t)
\end{array}\right]
$$

and $Y_{*}(t)=P(t) X(t)$, then $Y_{*}^{\prime}(t)=B(t) Y_{*}(t)$. Setting $Y(t)=Y_{*}(t) P(0)^{-1}$ we see that $Y^{\prime}(t)=B(t) Y(t)$ and $\left.Y(0)=P(0) X(0) P(0)\right)^{-1}=I$. Moreover, since $P(T)=P(0), Y(T)=P(T) X(T) P(T)^{-1}$. Since similar matrices have the same eigenvalues, it follows that the eigenvalues of $Y(T)$ are also equal to $\alpha_{1}$ and $\alpha_{2}$. If $Z(t)=R Y(t) R$, where

$$
R=\left[\begin{array}{rr}
1 & 0 \\
0 & -1
\end{array}\right]=R^{-1},
$$

then $Z^{\prime}(t)=C(t) Z(t), Z(0)=I$, where

$$
C(t)=R B(t) R=\left[\begin{array}{rr}
-b(t) u_{0}(t) & c(t) v_{0}(t) \\
e(t) u_{0}(t) & -f(t) v_{0}(t)
\end{array}\right] .
$$

To complete the proof of the Lemma we use the following result whose proof we take from [15]:

SUblemma: The elements of $Z(t)$ are strictly positive on the interval $0<t \leqslant T$.

Proof. Since the off-diagonal elements of $C(t)$ are strictly positive we may choose a constant $\gamma>0$ so large that $C(t)+\gamma I$ has strictly positive elements for $t \in[0, T]$. If $Z_{*}(t)=e^{\gamma t} Z(t)$, then $Z_{*}^{\prime}(t)=[C(t)+\gamma I] Z_{*}(t), Z_{*}(0)=I$, so

$$
Z_{*}(t)=I+\int_{0}^{t}[C(s)+\gamma I] Z_{*}(s) d s \text {. }
$$

Since $Z_{*}(t)$ is equal to the uniform limit on $(0, T]$ of the sequence $\left\{Z_{m}(t)\right\}_{m=0}^{\infty}$ defined inductively by $Z_{0}(t) \equiv I, Z_{m+1}(t)=I+\int_{0}^{t}[C(s)+\gamma I] Z_{m}(s) d s$, for $m$ $=1,2, \ldots$, and by induction the elements of each member of the sequence are nonnegative on $(0, T]$, the same is true of $Z_{*}(t)$. Since $Z_{*}(t)$ is never singular, it 
follows from the strict positivity of the elements of $C(t)+\gamma I$ and (3.3) that $Z_{*}(t)$ has strictly positive elements for $0<t \leqslant T$. Hence the same is true for $Z(t)$ and the proof is complete.

Continuing with the proof of Lemma 3.1 we note that $Z(T)=R^{-1} Y(T) R$ and $Y(T)$ have the same eigenvalues, namely $\alpha_{1}$ and $\alpha_{2}$. Since the elements of $Z(T)$ are strictly positive, it follows from the Perron-Frobenius theorem (see, for example [1] or [7]) that $Z(T)$ has a simple positive eigenvalue which is strictly greater than the modulus of the other eigenvalue and a corresponding eigenvector with strictly positive elements. Since, by Liouville's formula $\alpha_{1} \alpha_{2}=\operatorname{det} Z(T)=$ $\exp \int_{0}^{T} \operatorname{trace} C(s) d s, \alpha_{1}>0$ and $\alpha_{2}>0$ so we may assume $0<\alpha_{1}<\alpha_{2}$. To prove the lemma it is sufficient to prove that $\alpha_{2}<1$.

Let us note that from (2.2) and (2.3) we have that $a_{L}>c_{M} d_{M} / f_{L} \geqslant c_{M} d_{L} / f_{L}$ $>c_{M} e_{M} a_{M} / f_{L} b_{L} \geqslant c_{M} e_{M} a_{L} / f_{L} b_{L}$ and hence

$$
f_{L} b_{L}-c_{M} e_{M}>0 \text {. }
$$

Let $w=\operatorname{col}\left(w_{1}, w_{2}\right)$ be a constant vector which satisfies $Z(T) w=\alpha_{2} w, w_{1}>0$, $w_{2}>0$. Let $\theta(t)=Z(t) w$. If $\theta(t)=\operatorname{col}\left(\theta_{1}(t), \theta_{2}(t)\right)$, then by the sublemma $\theta_{1}(t)>$ and $\theta_{2}(t)>0$ for $0 \leqslant t \leqslant T$. Moreover, since $\theta^{\prime}(t)=C(t) \theta(t)$,

$$
\begin{aligned}
& \theta_{1}^{\prime}(t)=-b(t) u_{0}(t) \theta_{1}(t)+c(t) v_{0}(t) \theta_{2}(t), \\
& \theta_{2}^{\prime}(t)=e(t) u_{0}(t) \theta_{1}(t)-f(t) v_{0}(t) \theta_{2}(t) .
\end{aligned}
$$

Clearly,

$$
\begin{aligned}
& \theta_{1}^{\prime}(t) \leqslant-b_{L} u_{0}(t) \theta_{1}(t)+c_{M} v_{0}(t) \theta_{2}(t), \\
& \theta_{2}^{\prime}(t) \leqslant e_{M} u_{0}(t) \theta_{1}(t)-f_{L} v_{0}(t) \theta_{2}(t),
\end{aligned}
$$

and hence, by (3.4), $e_{M} \theta_{1}^{\prime}(t)+b_{L} \theta_{2}^{\prime}(t) \leqslant\left(e_{M} c_{M}-b_{L} f_{L}\right) \theta_{2}(t)<0$ for $0 \leqslant t \leqslant T$. It follows that $e_{M} \theta_{1}(T)+b_{L} \theta_{2}(T)<e_{M} \theta_{1}(0)+b_{L} \theta_{2}(0)$. But $\theta(T)=Z(T) w=$ $\alpha_{2} w=\alpha_{2} \theta(0)$ so $\theta_{1}(T)=\alpha_{2} \theta_{1}(0), \theta_{2}(T)=\alpha_{2} \theta_{2}(0)$. Hence $\alpha_{2}<1$ and by earlier remarks the lemma is proved.

\section{Proof of main theorem-first part}

THEOREM 1. Under the assumptions that $a(t), \ldots, f(t)$ are positive and T-periodic and (2.2) and (2.3) hold, the system (1.1) has a unique T-periodic solution $\operatorname{col}\left(u_{0}(t), v_{0}(t)\right)$ with $u_{0}(t)>0$ and $v_{0}(t)>0$ for all $t$. This solution is asymptotically stable. If $\operatorname{col}(u(t), v(t))$ is any solution with $u(0)>0$ and $v(0)>0$ then

$$
u(t)-u_{0}(t) \rightarrow 0, v(t)-v_{0}(t) \rightarrow 0 \text { as } t \rightarrow \infty .
$$


Proof. We shall first prove the existence of $\operatorname{col}\left(u_{0}(t), v_{0}(t)\right)$ among solutions with initial values in the rectangle

$$
S=\left\{(r, s) \mid 0<r<k_{1}, 0<s<k_{2}\right\},
$$

where $k_{1}$ and $k_{2}$ are defined as in the second section.

From degree theory (see, for example, [11]) we recall that if $\Omega \subset \mathbf{R}^{n}$ is open and bounded, if $g$ is an $\mathbf{R}^{n}$-valued function of class $C^{1}$ defined on the closure of $\Omega$ such that $g(x) \neq \overline{0}$ for all $x$ in the boundary of $\Omega$, and if $x \in \Omega$ and $g(x)=\overline{0}$ implies that the determinant of the Jacobian matrix of $g$ at $x$ denoted by $g^{\prime}(x)$ is nonzero, then $g(x)=\overline{0}$ has finitely many solutions $x_{1}, \ldots, x_{m}$ in $\Omega$ and

$$
\operatorname{deg}(g, \Omega, \overline{0})=\sum_{j=1}^{m} \operatorname{sgn} \operatorname{det} g^{\prime}\left(x_{j}\right) \text {. }
$$

If $D$ and $F$ are defined as in Lemma 2.3, $\left(r_{0}, s_{0}\right) \in D$ and $F\left(r_{0}, s_{0}\right)=\overline{0}$, then, by Lemma 3.1 , it follows that $\operatorname{det} F^{\prime}\left(r_{0}, s_{0}\right)$ is positive. Since, by Lemma 2.3 , $\operatorname{deg}(F, D, \overline{0})=1$, we see from formula (4.1) that there is a unique $\left(r_{0}, s_{0}\right) \in D$ such that $F\left(r_{0}, s_{0}\right)=0$. Thus, since $\delta>0$ can be taken arbitrarily small, it follows that there is a unique $\left(r_{0}, s_{0}\right) \in S$, such that $F\left(r_{0}, s_{0}\right)=\overline{0}$. Therefore, since the solution of (1.1) with initial value $\operatorname{col}(r, s)$ at $t=0$ is $T$-periodic if and only if $F(r, s)=\overline{0}$, we have shown that there is a unique positive, $T$-periodic solution of (1.1) which starts in $S$ at $t=0$.

As in the previous section we set

$$
\operatorname{col}\left(u_{0}(t), v_{0}(t)\right)=\operatorname{col}\left(u\left(t, r_{0}, s_{0}\right), v\left(t, r_{0}, s_{0}\right)\right) .
$$

As shown in the previous section, if $X(t)$ is the solution of the matrix differential equation (3.2) with $X(0)=I$ then the eigenvalues $\alpha_{1}$ and $\alpha_{2}$ of $X(T)$ satisfy $0<\alpha_{k}<1, k=1,2$. This means that the characteristic multipliers of the linear variational system corresponding to $\operatorname{col}\left(u_{0}(t), v_{0}(t)\right)$ have moduli less than 1 , so by the basic theory of differential equations the solution $\operatorname{col}\left(u_{0}(t), v_{0}(t)\right)$ of (1.1) is locally, asymptotically exponentially stable (see [3, p. 78-80, 321-322]). We now investigate the region of attraction.

Let $\operatorname{col}(u(t), v(t))$ be a solution of (1.1) such that $0<u(0)<k_{1}, 0<v(0)<k_{2}$. Choose $\delta>0$ such that $\delta<u(0), \delta<v(0)$, and (2.4) and (2.5) hold. Let $\operatorname{col}\left(u^{*}(t), v_{*}(t)\right)$ and $\operatorname{col}\left(u_{*}(t), v^{*}(t)\right)$ be as in the statement of Lemma 2.2. From Lemmas (2.1) and (2.2), it follows that for all $t \geqslant 0$ we have

$$
\begin{aligned}
u_{*}(t) & <u(t)<u^{*}(t), \\
v_{*}(t) & <v(t)<v^{*}(t), \\
\delta & \leqslant u_{*}(t)<u_{*}(t+T)<u^{*}(t+T)<u^{*}(t) \leqslant k_{1}, \\
\delta & \leqslant v_{*}(t)<v_{*}(t+T)<v^{*}(t+T)<v^{*}(t) \leqslant k_{2} .
\end{aligned}
$$

If for $t \geqslant 0$ and $m=1,2, \ldots$ we set

$$
u_{* m}(t)=u_{*}(t+m T), \quad v_{m}^{*}(t)=v^{*}(t+m T),
$$


then the sequence $\left\{u_{* m}(t)\right\}_{1}^{\infty}$ is increasing and bounded above on $[0, \infty)$, while $\left\{v_{m}^{*}(t)\right\}_{1}^{\infty}$ is decreasing and bounded below on $[0, \infty)$. Therefore there exist functions $\bar{u}(t)$ and $\bar{v}(t)$ defined on $[0, \infty)$ such that

$$
\lim _{m \rightarrow \infty} u_{* m}(t)=\bar{u}(t), \quad \lim _{m \rightarrow \infty} v_{m}^{*}(t)=\bar{v}(t) \text { for } t \geqslant 0 .
$$

Since for each $m=1,2, \ldots \operatorname{col}\left(u_{* m}(t), v_{m}^{*}(t)\right)$ is a solution of (1.1) (by $T$-periodicity in $t$ of the right-hand sides), we see that the derivatives of the members of the sequences $\left\{u_{*_{m}}(t)\right\}_{m=1}^{\infty}$ and $\left\{v_{m}^{*}(t)\right\}_{m=1}^{\infty}$ are bounded on $[0, \infty)$. Hence by Ascoli's lemma, for any compact subinterval of $[0, \infty)$, there exist subsequences of these two sequences which converge uniformly to $\bar{u}(t)$ and $\bar{v}(t)$ on this subinterval. Hence, $\bar{u}$ and $\bar{v}$ are continuous, so by Dini's theorem and monotonicity of the two sequences, we see that the convergence in (4.6) is uniform on compact subintervals of $[0, \infty)$. Since $\operatorname{col}\left(u_{*_{m}}(t), v_{m}^{*}(t)\right)$ is a solution of (1.1) for $m=$ $1,2, \ldots$, it follows that the derivatives of members of the sequences $\left\{u_{* m}(t)\right\}_{1}^{\infty}$ and $\left\{v_{m}^{*}(t)\right\}$ converge uniformly on compact subintervals of $[0, \infty)$. Hence, $\bar{u}(t)$ and $\bar{v}(t)$ have continuous derivatives and $\operatorname{col}(\bar{u}(t), \bar{v}(t))$ is a solution of system (1.1). Moreover, $\bar{u}(t+T)=\lim _{m \rightarrow \infty} u_{* m}(t+T)=\lim _{m \rightarrow \infty} u_{*, m+1}(t)=\bar{u}(t)$. Similarly, $\bar{v}(t+T)=\bar{v}(t)$ for $t \geqslant 0$. It follows that $\bar{u}(t)$ and $\bar{v}(t)$ can be periodically extended to $(-\infty, \infty)$ so that $\operatorname{col}(\bar{u}(t), \bar{v}(t))$ is a $T$-periodic solution of (1.1). From (4.4) and (4.5), we see that $\delta<\bar{u}(0)<k_{1}$ and $\delta<\bar{v}(0)<k_{2}$. Therefore, since col $\left(u_{0}(t), v_{0}(t)\right)$ is the unique $T$-periodic solution of (1.1) with $(u(0), v(0)) \in S$, we see that $\bar{u}(t) \equiv u_{0}(t), \bar{v}(t) \equiv v_{0}(t)$.

We now show that $u_{*}(t)-u_{0}(t) \rightarrow 0, v^{*}(t)-v_{0}(t) \rightarrow 0$ as $t \rightarrow \infty$. Since (4.6) holds uniformly for $0 \leqslant t \leqslant T$, given $\varepsilon>0$ there exists $N$ such that if $m \geqslant N$, then $\left|u_{* m}(s)-u_{0}(s)\right|<\varepsilon,\left|v_{m}^{*}(s)-v_{0}(s)\right|<\varepsilon$ for $0 \leqslant s \leqslant T$. If $t \geqslant(N+1) T$ then $t=m T+s$ for some $m \geqslant N$ and some $s \in[0, T)$ and consequently, by $T$-periodicity of $u_{0},\left|u_{*}(t)-u_{0}(t)\right|=\left|u_{* m}(s)-u_{0}(s)\right|<\varepsilon$. Similarly, $\mid v_{*}(t)-$ $v_{0}(t) \mid<\varepsilon$ if $t \geqslant(N+1) T$ and the claim is established.

A repetition of the same reasoning applied to the solution $\operatorname{col}\left(u^{*}(t), v_{*}(t)\right)$ instead of $\operatorname{col}\left(u_{*}(t), v^{*}(t)\right)$ shows that $u^{*}(t)-u_{0}(t) \rightarrow 0, v_{*}(t)-v_{0}(t) \rightarrow 0$ as $t \rightarrow \infty$. Therefore, by (4.2) and (4.3) we see that $u(t)-u_{0}(t) \rightarrow 0, v(t)-v_{0}(t)$ $\rightarrow 0$ as $t \rightarrow \infty$.

We remove the restriction that $(u(0), v(0)) \in S$ in the next section.

\section{Global asymptotic stability}

To complete the proof of the main theorem we use a result concerning the logistic equation

$$
U^{\prime}(t)=U(t)[A(t)-B(t) U(t)]
$$


where it is assume that $A$ and $B$ are continuous $T$-periodic functions with $A(t)>0$ and $B(t)>0$ for all $t$. The following result can be derived from a study of more general functional equations given in [5] but for completeness we give a much shorter alternate proof.

LemMa 5.1. There exists a unique positive T-periodic solution $U_{0}(t)$ of (5.1). If $U(t)$ is any other solution of (5.1) with $U(0)>0$, then

$$
U(t)-U_{0}(t) \rightarrow 0 \text { as } t \rightarrow \infty \text {. }
$$

Proof. If $\varepsilon>0$ is so small that

$$
\varepsilon[A(t)-\varepsilon B(t)]>0
$$

for all $t$, and $U_{1}(t)$ is the solution with $U_{1}(0)=\varepsilon$, then $U_{1}(t)>\varepsilon$ for $t>0$ so $U_{1}(T)>U_{1}(0)$. Similarly, if $k>\varepsilon$ is chosen so that $k[A(t)-k B(t)]<0$, then the solution $U_{2}(t)$ with $U_{2}(0)=k$ satisfies $U_{2}(T)<U_{2}(0)$. Hence, there exists $c$ in $(\varepsilon, k)$ such that if $U_{0}(t)$ is the solution with $U_{0}(0)=c$, then $U_{0}(T)=U_{0}(0)$ so $U_{0}(t)$ is $T$-periodic and positive (since 0 is a solution of (5.1)). If $U(t)$ is any positive solution of (5.1) and we set $W(t)=1 / U(t), W_{0}(t)=1 / U_{0}(t)$ then $W^{\prime}(t)-W_{0}^{\prime}(t)=-A(t)\left[W(t)-W_{0}(t)\right]$. Hence, $W(t)-W_{0}(t)=$ constant $(\exp$ $\left.-\int_{0}^{t} A(s) d s\right)$ so $W(t)-W_{0}(t) \rightarrow 0$ as $t \rightarrow \infty$. This implies that $U(t)-U_{0}(t) \rightarrow$ 0 as $t \rightarrow \infty$ and the lemma is proved.

LEMMA 5.2. If $U_{0}(t)$ is the unique positive T-periodic solution of

$$
U^{\prime}(t)=U(t)[a(t)-b(t) U(t)] \text { and } V_{0}(t) \text { is the }
$$

unique positive $T$-periodic solution of

$$
V^{\prime}(t)=V(t)[d(t)-f(t) V(t)],
$$

then

$$
U_{0}(t) \leqslant a_{M} / b_{L}, \quad V_{0}(t) \leqslant d_{M} / f_{L} .
$$

Proof. If $U_{0}(t)$ attains its maximum when $t=t_{1}$, then since $U^{\prime}\left(t_{1}\right)=0, U\left(t_{1}\right)$ $=a\left(t_{1}\right) / b\left(t_{1}\right) \leqslant a_{M} / b_{L}$. The proof of the second inequality is similar.

LEMMA 5.3. Let col $(u(t), v(t))$ be a solution of the system (1.1) such that $u(0)>0$ and $v(0)>0$. If $U(t)$ and $V(t)$ satisfy the scalar differential equations (5.2) and (5.3) respectively and $u(0)=U(0), v(0)=V(0)$, then $u(t) \leqslant U(t)$, $v(t) \leqslant V(t)$ for all $t \geqslant 0$.

Proof. Since $\operatorname{col}(U(t), 0)$ and $\operatorname{col}(0), V(t))$ are both solutions of the system (1.1), the assertion of the lemma follows from Lemma 2.1.

COMPLETION OF PROOF OF THEOREM 1: As in section 4, let $S$ denote the open rectangle in the $u, v$-plane given by $0<u<k_{1}, 0<v<k_{2}$. It has been shown 
that if $\operatorname{col}(u(t), v(t))$ is a solution of (1.1) with $(u(0), v(0)) \in S$ then $u(t)-u_{0}(t)$ $\rightarrow 0$ and $v(t)-v_{0}(t) \rightarrow 0$ as $t \rightarrow \infty$. We shall show that if $\operatorname{col}(u(t), v(t))$ is any solution of (1.1) with $u(0)>0$ and $v(0)>0$, then there exists an integer $m \geqslant 1$ such that $(u(m T), v(m T)) \in S$. By $T$-periodicity of the system, $\operatorname{col}(\bar{u}(t), \bar{v}(t))=$ $\operatorname{col}(u(t+m T), v(t+m T))$ is a solution in $S$ at $t=0$. From this and $T$-periodicity of $\operatorname{col}\left(u_{0}(t), v_{0}(t)\right)$ it will follow that

$$
\begin{array}{ll}
u(t+m T)-u_{0}(t+m T) \rightarrow 0 & \text { as } t \rightarrow \infty \\
v(t+m T)-v_{0}(t+m T) \rightarrow 0 & \text { as } t \rightarrow \infty,
\end{array}
$$

so $u(t)-u_{0}(t) \rightarrow 0, v(t)-v_{0}(t) \rightarrow 0$, as $t \rightarrow \infty$ and the proof of Theorem 1 will be complete.

Suppose then that $\operatorname{col}(u(t), v(t))$ is a solution of (1.1) with $u(0)>0$ and $v(0)>0$. If $U(t)$ and $V(t)$ are the solutions of (5.2) and (5.3) respectively such that $u(0)=U(0)$ and $v(0)=V(0)$, then by Lemma 5.3, $u(t) \leqslant U(t)$ and $v(t) \leqslant$ $V(t)$ for $t \geqslant 0$. From Lemma 5.1. $U(t)-U_{0}(t) \rightarrow 0$ and $V(t)-V_{0}(t) \rightarrow 0$ as $t \rightarrow \infty$ where $U_{0}(t)$ and $V_{0}(t)$ are the unique positive $T$-periodic solutions of (5.2) and (5.3) respectively. Since $U_{0}(t) \leqslant a_{M} / b_{L}<k_{1}$ and $V_{0}(t) \leqslant d_{M} / f_{L}<k_{2}$, for all $t$, it follows that for $t$ sufficiently large and positive $u(t) \leqslant U(t)<k_{1}$ and $v(t) \leqslant V(t) \leqslant k_{2}$. In particular, there exists an integer $m \geqslant 1$ such that $(u(m T), v(m T)) \in S$. By earlier remarks, this complete the proof of Theorem 1 .

\section{Upper and lower bounds}

In this section, through very elementary means, we establish upper and lower bounds for the components of the unique periodic solution of the system (1.1) under conditions (2.2) and (2.3).

THEOREM 2. If $\operatorname{col}\left(u_{0}(t), v_{0}(t)\right)$ is the unique positive periodic solution of system (1.1) whose existence was established in Theorem 1 , then for $t \in(-\infty, \infty)$

$$
\begin{aligned}
& \frac{a_{L} f_{L}-c_{M} d_{M}}{b_{M} f_{L}-c_{M} e_{L}} \leqslant u_{0}(t) \leqslant \frac{a_{M} f_{M}-c_{L} d_{L}}{b_{L} f_{M}-c_{L} e_{M}}, \\
& \frac{b_{L} d_{L}-e_{M} a_{M}}{b_{L} f_{M}-c_{L} e_{M}} \leqslant v_{0}(t) \leqslant \frac{b_{M} d_{M}-e_{L} a_{L}}{b_{M} f_{L}-c_{M} e_{L}} .
\end{aligned}
$$

Proof. From (3.4) we have $b_{L} f_{M}-c_{L} e_{M} \geqslant b_{L} f_{L}-c_{M} e_{M}>0$, and $b_{M} f_{L}-$ $c_{M} e_{L} \geqslant b_{L} f_{L}-c_{M} e_{M}>0$.

For brevity we use $u_{M}, u_{L}, v_{M}$ and $v_{L}$ instead of $u_{0 M}, u_{0 L}, v_{0 M}$, and $v_{0 L}$ to denote the maximum values of $u_{0}(t)$ and the maximum and minimum values of $v_{0}(t)$ respectively. If $u_{M}=u_{0}\left(t_{1}\right)$ and $v_{L}=v_{0}\left(t_{2}\right)$ then $u_{0}^{\prime}\left(t_{1}\right)=v_{0}^{\prime}\left(t_{2}\right)=0$, so 
from (1.1)

$$
\begin{aligned}
& 0=a\left(t_{1}\right)-b\left(t_{1}\right) u_{0}\left(t_{1}\right)-c\left(t_{1}\right) v_{0}\left(t_{1}\right) \\
& 0=d\left(t_{2}\right)-e\left(t_{2}\right) u_{0}\left(t_{2}\right)-f\left(t_{2}\right) v_{0}\left(t_{2}\right)
\end{aligned}
$$

Hence

$$
\begin{aligned}
b_{L} u_{M} & \leqslant b\left(t_{1}\right) u_{M}=a\left(t_{1}\right)-c\left(t_{1}\right) v_{0}\left(t_{1}\right) \\
& \leqslant a_{M}-c_{L} v_{L}, \\
f_{M} v_{L} & \geqslant f\left(t_{2}\right) v_{L}=d\left(t_{2}\right)-e\left(t_{2}\right) u_{0}\left(t_{2}\right) \\
& \geqslant d_{L}-e_{M} u_{M},
\end{aligned}
$$

so

$$
u_{M}=\left[a\left(t_{1}\right)-c\left(t_{1}\right) v_{0}\left(t_{1}\right)\right] / b\left(t_{1}\right) \leqslant a_{M} / b_{L}-c_{L} v_{L} / b_{L}
$$

and

$$
v_{L}=\left[d\left(t_{2}\right)-e\left(t_{2}\right) u_{0}\left(t_{2}\right)\right] / f\left(t_{2}\right) \geqslant d_{L} / f_{M}-e_{M} u_{M} / f_{M} .
$$

Substituting (6.4) in (6.3) we obtain

$$
u_{M} \leqslant \frac{a_{M} f_{M}-c_{L} d_{L}}{b_{L} f_{M}}+\frac{c_{L} e_{M}}{b_{L} f_{M}} u_{M},
$$

from which it follows that

$$
u_{M} \leqslant \frac{a_{M} f_{M}-c_{L} d_{L}}{b_{L} f_{M}-c_{L} e_{M}}
$$

Similarly, substituting (6.3) into (6.4) we obtain

$$
v_{L} \geqslant \frac{b_{L} d_{L}-e_{M} a_{M}}{b_{L} f_{M}-c_{L} e_{M}}
$$

We obtain the same two differential equations in the system (1.1) when we interchange $u$ with $v, a$ with $d, b$ with $f$, and $c$ with $e$. Therefore by (6.5), (6.6), and duality, we obtain

$$
v_{M} \leqslant \frac{d_{M} b_{M}-e_{L} a_{L}}{f_{L} b_{M}-e_{L} c_{M}}
$$

and

$$
u_{L} \geqslant \frac{f_{L} a_{L}-c_{M} d_{M}}{f_{L} b_{M}-e_{L} c_{M}}
$$


The inequalities (6.1) and (6.2) follow from (6.5)-(6.8) and the theorem is proved.

\section{Diffusion}

Let $\Omega$ be a smooth bounded domain in $\mathbf{R}^{N}$. We consider the system of parabolic differential equations

$$
\begin{aligned}
& u_{t}=K_{1}(t) \Delta u+u[a(t)-b(t) u-c(t) v] \\
& v_{t}=K_{2}(t) \Delta v+v[d(t)-e(t) u-f(t) v]
\end{aligned}
$$

subject to the boundary conditions

$$
\frac{\partial u}{\partial n}\left|\partial \Omega \times \mathbf{R}=\frac{\partial v}{\partial n}\right| \partial \Omega \times \mathbf{R}=0,
$$

where $\partial / \partial n$ denotes differentiation in the direction of the outer normal to the boundary of the cylinder $\bar{\Omega} \times \mathbf{R}$. In [2] Brown showed that if $K_{1}, K_{2}, a, \ldots, f$ are positive constants and (1.5) holds then if $\operatorname{col}(u(x, t), v(x, t))$ satisfy (7.1) and (7.2) for $(x, t) \in \bar{\Omega} \times(0, \infty)$,

$$
\begin{gathered}
u, v \in C(\bar{\Omega} \times[0, \infty)) \cap C^{2,1}(\bar{\Omega} \times(0, \infty)), \\
u(x, 0) \geqslant 0, \quad v(x, 0) \geqslant 0, \quad u(x, 0) \not \equiv 0, \quad v(x, 0) \not \equiv 0,
\end{gathered}
$$

then $u(x, t) \rightarrow u_{0}, v(x, t) \rightarrow v_{0}$ as $t \rightarrow \infty$ uniformly with respect to $x \in \bar{\Omega}$, where $u_{0}=(a f-d c) / D, v_{0}=(b d-a e) / D, D=b f-c e$. In [4], the second author and C. Cosner showed that if $K_{1}, K_{2}, a$, and $d$ are continuous positive $T$-periodic functions, $b, c, d$, and $f$ are positive constants, and (2.2) and (2.3) (Gopalsamy's conditions) hold, then for any solution $\operatorname{col}(u(x, t), v(x, t))$ of the initial value problem satisfying (7.4) and

$$
\begin{array}{ll}
u(x, 0)<a_{M} / b, & v(x, 0)<d_{M} / F, \\
u(x, t) \rightarrow u_{0}(t), & v(x, t) \rightarrow v_{0}(t),
\end{array}
$$

uniformly with respect to $x \in \bar{\Omega}$, where $\operatorname{col}\left(u_{0}(t), v_{0}(t)\right)$ is the unique positive $T$-periodic solution of (1.1).

Using essentially the same argument as in Theorem 2.1 of [4], one can prove

THEOREM 3. If $K_{1}, K_{2}, a, \ldots, f$ are continuous, positive, $T$-periodic functions satisfying (2.2) and (2.3) and $\operatorname{col}(u(x, t), v(x, t))$ is any solution of the initial value problem corresponding to (7.1) and (7.2) such that $u(x, 0)$ and $v(x, 0)$ are 
sufficiently regular, (7.4) holds and $u(x, 0)<a_{M} / b_{L}, v(x, 0)<d_{M} / f_{L}$ for $x$ in $\Omega$ then (7.6) holds uniformly with respect to $x \in \Omega$ where $\operatorname{col}\left(u_{0}(t), v_{0}(t)\right)$ is the unique, positive, $T$-periodic solution of (1.1).

\section{References}

[1] R. E. Bellman, Introduction to matrix analysis (McGraw-Hill, New York, 1960).

[2] P. N. Brown, "Decay to uniform states in ecological interactions," SIAM J. Appl. Math. 38 (1980), 22-37.

[3] E. A. Coddington and N. Levinson, Theory of ordinary differential equations (McGraw-Hill, New York, 1955).

[4] C. Cosner and A. C. Lazer, "Stable co-existence states in the Lotka-Volterra competition model with diffusion," SIAM J. Appl. Math. 44 (1984), 1112-32.

[5] J. M. Cushing, "Stable positive periodic solutions of time-dependent logistic equation under possible hereditary influences," J. Math. Anal. Appl. 60 (1970), 747-754.

[6] J. M. Cushing, "Two species competition in a periodic environment," J. Math. Blol. 10 (1980), $385-400$.

[7] F. R. Gantmacher, Applications of the theory of matrices (Interscience, New York, 1959).

[8] K. Golpalsamy, "Exchange of equilibria in two species Lotka-Volterra competition models," $J$. A ustral. Math. Soc. Ser. B 24 (1982), 160-170.

[9] K. Gopalsamy, "Global asymptotic stability in a periodic Lotka-Volterra system," $J$. Austral. Math. Soc. Ser. B 27 (1985), 66-72.

[10] J. K. Hale, Ordınary differential equations (Wiley-Interscience, New York, 1969).

[11] N. G. Lloyd, Degree theory (Cambridge University Press, 1978).

[12] P. de Mottoni and A. Schiaffino, "Competition system with periodic coefficients: A geometry approach," J. Math. Biol. 11 (1981), 319-335.

[13] J. M. Smith, Mathematical ideas in biology (Cambridge University Press, 1968).

[14] J. T. Schwartz, Nonlinear functional analysis (Gordon and Breach, New York, 1969).

[15] A. D. Ziebur, "New directions in linear differential equations," SIAM Rev. 21 (1979), 57-70. 